\title{
Self-assembly of silver nanoparticles on chitosan/polyvinylpyrrolidone modified micro-needle electrode for amperometric detection of nitrate in seawater
}

\author{
Jie Wang ${ }^{\mathrm{a}}$, Ying $\mathrm{Li}^{\mathrm{a}}$, Dawei Pan ${ }^{\mathrm{b}, \mathrm{c}, \mathrm{d}}$, Haitao Han ${ }^{\mathrm{b}, \mathrm{c}, \mathrm{d}, *}$, Peiqing Zhang ${ }^{\mathrm{a}, *}$ \\ ${ }^{a}$ School of Chemistry and Chemical Engineering, Yantai University, Yantai 264005, People's Republic of China \\ ${ }^{\mathrm{b}}$ CAS Key Laboratory of Coastal Environmental Processes and Ecological Remediation, Shandong Key Laboratory of Coastal Environmental Processes, Research Center \\ for Coastal Environment Engineering Technology of Shandong Province, Yantai Institute of Coastal Zone Research, Chinese Academy of Sciences, Yantai 264003, \\ People's Republic of China \\ ${ }^{\mathrm{c}}$ Center for Ocean Mega-Science, Chinese Academy of Sciences, Qingdao 266071, People's Republic of China \\ ${ }^{\mathrm{d}}$ University of Chinese Academy of Sciences, Beijing 100049, People's Republic of China
}

\section{A R T I C L E I N F O}

\section{Keywords:}

Micro-needle electrode

Silver nanoparticles

Chitosan

Polyvinylpyrrolidone

Nitrate

Seawater

\begin{abstract}
A B S T R A C T
In this work, silver nanoparticles (AgNPs) were self-assembled on the micro-needle electrode (MNE) with the help of chitosan (CTS) and polyvinylpyrrolidone (PVP) and used for the amperometric (i-t) determination of nitrate $\left(\mathrm{NO}_{3}^{-}\right)$in seawater samples. CTS/PVP was electrodeposited on the MNE surface to improve the conductivity and specific surface area of the MNE, and to provide reduction conditions needed for $\mathrm{Ag}^{+}$reduction and strong binding affinity for combination of AgNPs which had excellent catalytic activity for $\mathrm{NO}_{3}^{-}$. AgNPs were selfassembled uniformly on the surface of MNE with the diameter of about 150-400 nm. Thanks to the unique aciform structure of MNE, the outstanding adhesion ability of CTS/PVP, and the excellent electrocatalytic ability of AgNPs, the so-prepared AgNPs/CTS/PVP/MNE showed significantly improved performance and stability for the amperometric determination of $\mathrm{NO}_{3}^{-}$. Under the optimal conditions, the AgNPs/CTS/PVP/MNE was found to have a wide linear range $(5-2000 \mu \mathrm{M})$ and a low limit of detection $(1.2 \mu \mathrm{M})$. Furthermore, the AgNPs/CTS/PVP/ MNE was successfully used for the direct determination of $\mathrm{NO}_{3}^{-}$in coastal seawater samples with satisfactory results.
\end{abstract}

\section{Introduction}

Nitrates $\left(\mathrm{NO}_{3}^{-}\right)$are naturally present in soil, water, and food, which has important effects on environmental and human health $[1,2]$. In marine environment, the photosynthesis of phytoplankton is largely controlled by $\mathrm{N}$ nutrients including $\mathrm{NO}_{3}^{-}$, nitrite, and ammonium [3]. $\mathrm{NO}_{3}^{-}$is of particular interest because it represents the main source of $\mathrm{N}$ in marine ecosystems and acts as a marker for water quality [4]. The seawater environment with excessive $\mathrm{NO}_{3}^{-}$concentration will break the balance of local marine ecosystems, leading to hyper proliferation of algae, generation of red tides, deterioration of water quality, and destruction of biodiversity [5]. Moreover, the concentration of $\mathrm{NO}_{3}^{-}$is easy to change with the external environment. Therefore, to understand the $\mathrm{N}$ cycle and dynamics of marine ecosystems, it is necessary to determine the concentration of $\mathrm{NO}_{3}^{-}$in seawater rapidly and accurately.

To date, a wide range of analytical methods have been developed for determination of $\mathrm{NO}_{3}^{-}$. The most commonly used techniques include ionexchange chromatography [6], capillary electrophoresis [7], colorimetry [8], UV spectroscopy [9], reflectance mid-infrared spectroscopy [10], and high-performance liquid chromatography [11]. Although many of these methods are sensitive and accurate, most of them require sample pretreatment and/or pre-concentration which are relatively time-consuming, fiddly and costly [12]. Electrochemical methods represent an interesting alternative with the advantages of low cost, ease of use, low energy requirements and simple procedures [13]. Furthermore, it presents the possibility to build portable systems that are

\footnotetext{
* Corresponding authors at: CAS Key Laboratory of Coastal Environmental Processes and Ecological Remediation, Shandong Key Laboratory of Coastal Envi-

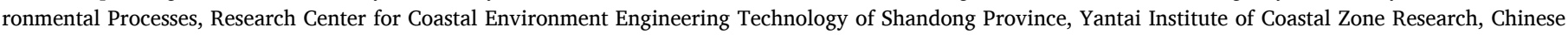

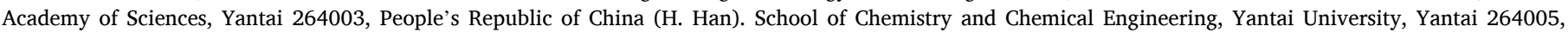
People's Republic of China (P. Zhang).

E-mail addresses: hthan@yic.ac.cn (H. Han), zhangpqytu@126.com (P. Zhang).
} 


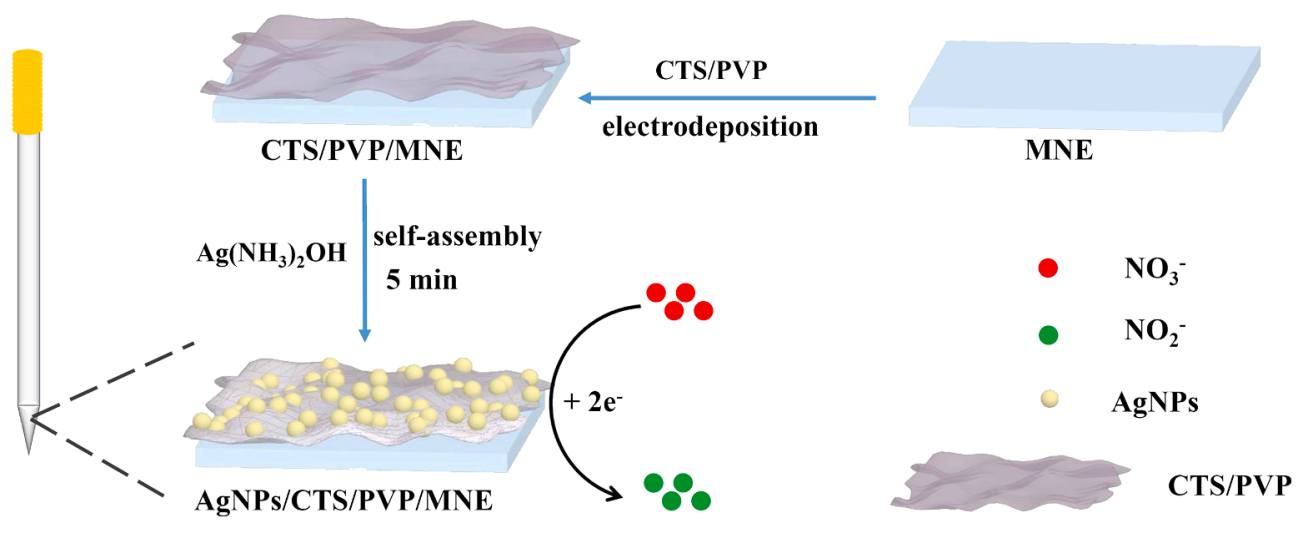

Scheme 1. Schematic illustration for the fabrication procedure of AgNPs/CTS/PVP/MNE.

compatible either for laboratory or on-site measurements. Recently, many electrochemical sensors based on different electrodes have been prepared for the determination of $\mathrm{NO}_{3}^{-}$. Different electrode materials including copper [14,15], precious metals [16], bimetallic alloys $[17,18]$, and base metals [19-21] have been investigated for voltammetric and amperometric analysis of $\mathrm{NO}_{3}^{-}$. For example, the $\mathrm{Cu}$ microsphere modified electrode presented in our previous work could be successfully used for the determination of $\mathrm{NO}_{3}^{-}$in river water samples [12]. However, these nanomaterials-based electrodes are usually applied in the environment with $\mathrm{pH}$ value below 3.0. Obviously, it is not suitable for the direct detection of $\mathrm{NO}_{3}^{-}$in natural seawater with $\mathrm{pH}$ about 8.0. Therefore, there is still an urgent need for novel and effective electrodes for direct detection $\mathrm{NO}_{3}^{-}$in seawater.

$\mathrm{Ag}$ nanoparticles (AgNPs), one of the popular metal nanomaterials, have several advantages such as small size, large surface area, high surface activity and excellent catalytic performance, and have been widely assembled on electrodes to act as electro-catalytic material for various reactions [22-24]. Importantly, AgNPs also exhibit an extremely fast response for the electrochemical reduction of $\mathrm{NO}_{3}^{-}$it in natural water with no need to change the $\mathrm{pH}$ value [25]. Acupuncture which is considered as a pivotal component of Eastern medicine has the functions of relieving pain, warming the meridians and promoting the blood flow through the application of an acupuncture needle into the physical body of the patient $[26,27]$. The stainless steel acupuncture needle has the properties of conductivity, small size, large specific surface area, and easy operation, which makes it a potential electrode material for electrochemical sensing applications. Therefore, micro-needle electrode (MNE) made of stainless steel acupuncture needle provides a promising electrochemical sensing platform with large specific surface area for the combination of functional nanomaterials for different small biomolecules and inorganic ions $[28,29]$. To achieve the modification of AgNPs on MNE, surface modification is clearly needed because of the smooth surface of stainless steel MNE [24].

As a natural biopolymer, Chitosan (CTS) exhibits excellent properties such as film-forming ability, biocompatibility, multiple functional groups, and pH-dependent solubility in aqueous media [30]. As reported, CTS can form stable membrane with conducting materials and enhance their conductivity [31,32]. Many kinds of materials such as graphene oxide (GO), carbon nanotubes (CNTs), $\mathrm{Ag}, \mathrm{Si}$, and $\mathrm{Cu}$ have ever been reported to combine with CTS due to its reducibility, abundant functional groups, and film-forming ability. These properties make CTS a widely used immobilization agent for different nanomaterials to fabricate novel electrochemical sensors. Moreover, the functional groups of CTS can reduce $\mathrm{Ag}^{+}$to $\mathrm{Ag}^{0}$, which looks like Tollen reaction and provides conditions for the self-assembly of AgNPs on the electrode $[33,34]$. Polyvinylpyrrolidone (PVP) is a well-known macromolecule surfactant with advantages of low toxicity, biocompatibility, high surface activity, and strong adsorption ability [35]. In addition, it is known that PVP is one of the attractive polymers to immobilize inorganic nanoparticles due to its strong affinity of pyridyl group to metals and ability to undergo hydrogen bonding with polar species [36]. Furthermore, the composite of CTS and PVP (CTS/PVP) can not only protect the electrode, but also enhance the bonding ability with metal nanomaterials $[37,38]$.

In this work, AgNPs were self-assembled on the MNE which was surface modified with CTS/PVP to fabricate the AgNPs/CTS/PVP/MNE for amperometric determination of $\mathrm{NO}_{3}^{-}$in seawater. As the conductive polymer, reducing agent, and adhesion agent, CTS/PVP film offered a lot of binding sites for the combination of AgNPs which had excellent catalytic performance for the electrochemical reduction of $\mathrm{NO}_{3}^{-}$. The soprepared AgNPs/CTS/PVP/MNE exhibited outstanding performance for the amperometric detection of $\mathrm{NO}_{3}^{-}$. Furthermore, the AgNPs/CTS/ $\mathrm{PVP} / \mathrm{MNE}$ was successfully used for the direct detection of $\mathrm{NO}_{3}^{-}$in coastal seawater samples at natural $\mathrm{pH}$ condition.

\section{Experimental}

\subsection{Materials and reagents}

Silver nitrate $\left(\mathrm{AgNO}_{3}\right)$, sodium nitrate $\left(\mathrm{NaNO}_{3}\right)$, ethanol $\left(\mathrm{CH}_{3} \mathrm{CH}_{2} \mathrm{OH}\right)$, acetic acid (HAc), ammonium hydroxide, PVP, and CTS were purchased from Sinopharm Chemical Reagent Co. Ltd. (Shanghai, China). Unless otherwise stated, phosphate buffer ( $\mathrm{pH}$ 7.0) was used as the supporting electrolyte for electrochemical experiments. Deionized water (18.2 $\mathrm{M} \Omega \mathrm{cm}$ specific resistance) obtained from Pall Cascada laboratory water system was used throughout. All chemicals were analytical reagents and used without further purification. Stainless steel acupuncture needles (Suzhou Medical Appliance Factory Co., Ltd, China) were used as the electrode substrates. Silicone rubber purchased from Liyang Kangda Chemical Co. Ltd., China was adopted as the sealant.

\subsection{Apparatus}

The morphologies of the bare and modified MNEs were characterized by scanning electron microscopy (SEM, Hitachi S-4800 microscope, Japan). X-ray photoelectron spectroscopy (XPS) was carried out on a Thermo ESCALAB 250XI (America). All the electrochemical experiments including cyclic voltammetry (CV) and amperometric i-t were performed on a CHI660E electrochemical workstation (CH Instruments, Inc., Shanghai, China). The modified MNE served as the working electrode, with $\mathrm{Ag} / \mathrm{AgCl}(3.0 \mathrm{M} \mathrm{KCl})$ and platinum foil serving as the reference and counter electrode, respectively. All potentials were measured with respect to the $\mathrm{Ag} / \mathrm{AgCl}$ reference electrode.

\subsection{Preparation of the AgNPs/CTS/PVP/MNE}

The procedure for the fabrication of AgNPs/CTS/PVP/MNE was 

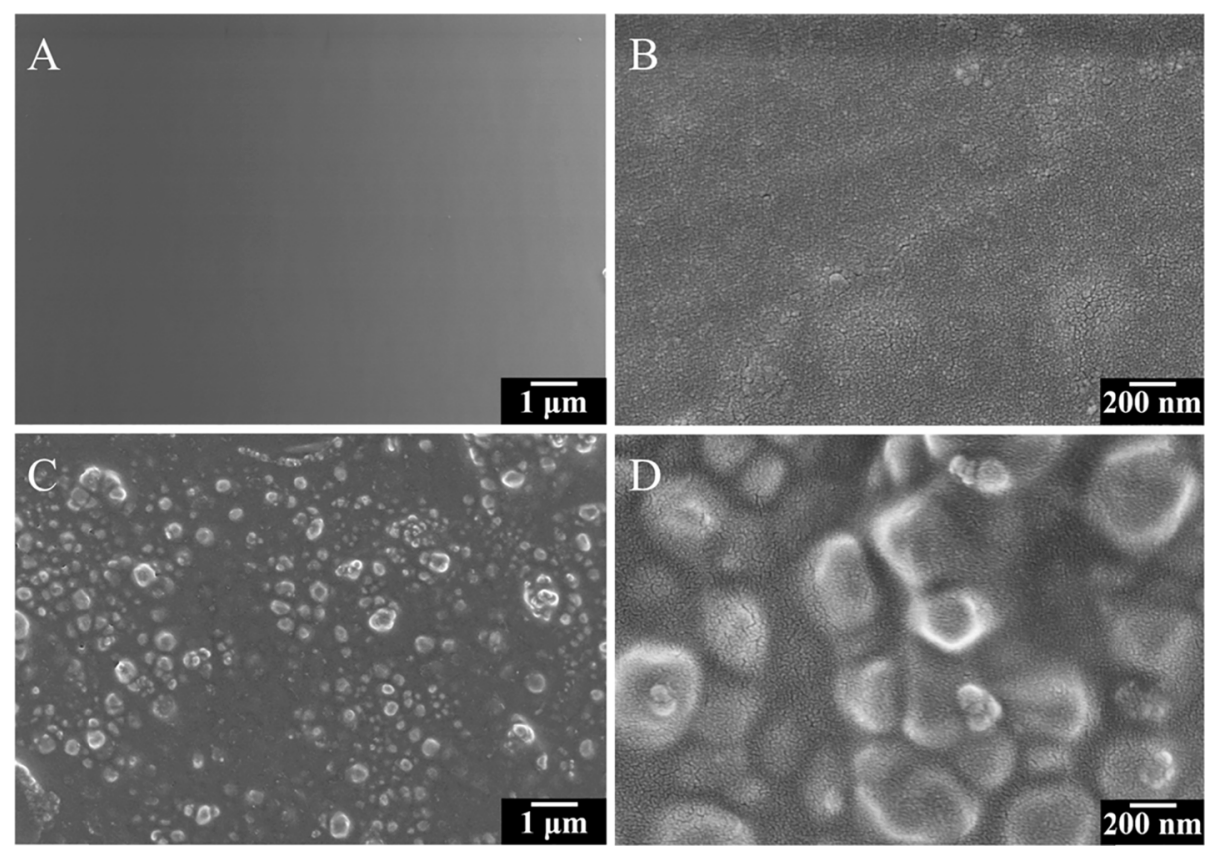

Fig. 1. SEM images of the MNE (A), CTS/PVP/MNE (B), and AgNPs/CTS/PVP/MNE (C, D).

illustrated in Scheme 1. Firstly, the MNE was manufactured by stainless steel acupuncture needles as described in our previous works [12,39]. Simply, the needle body part of the acupuncture needle was insulated and sealed with silicone rubber; leaving the needle tip about $1 \mathrm{~mm}$ and needle handle out for the sensing surface and electrode wire, respectively. Secondly, the cleaning of the stainless steel acupuncture needle (diameter of $0.25 \mathrm{~mm}$, length of $60 \mathrm{~mm}$ ) was conducted with the help of sonication in ethanol and deionized water for $5 \mathrm{~min}$, respectively. Then the CTS/PVP film was deposited on the MNE surface to prepare the CTS/ PVP modified MNE (CTS/PVP/MNE) by electrodepositing in the mixed solution of $0.1 \mathrm{mg} / \mathrm{ml} \mathrm{PVP}$ and CTS ( $0.1 \mathrm{M} \mathrm{HAc}$ ) at the potential of $-2 \mathrm{~V}$ for $200 \mathrm{~s}$. Lastly, the CTS/PVP/MNE was put into the $\mathrm{AgNO}_{3}$ solution for 5 min to achieve the self-assembly of AgNPs on the CTS/PVP/MNE. After careful cleaning and drying at room temperature, the AgNPs/CTS/ $\mathrm{PVP} / \mathrm{MNE}$ was obtained eventually.

\subsection{Electrochemical analysis procedure}

The CV plots were obtained in PBS solution (pH 7) in the range of -0.8 to $0.4 \mathrm{~V}$ at the scan rate of $100 \mathrm{mV} / \mathrm{s}$. The current responses of $\mathrm{NO}_{3}^{-}$ on different MNEs were investigated by amperometric i-t technique using the following parameters: potential of $-1.1 \mathrm{~V}$, pulse period of 0.5 $\mathrm{s}$, and quiet time of $2 \mathrm{~s}$.

\section{Results and discussion}

\subsection{Characterization of the AgNPs/CTS/PVP/MNE}

To investigate the surface morphology of AgNPs/CTS/PVP/MNE, SEM characterization was conducted (Fig. 1). It can be seen clearly that the surface of the bare MNE was very smooth (Fig. 1A). It has been described in our previous works that the smooth surface provided few binding sites for functional nanomaterials [39]. As shown in Fig. 1B, a uniform and dense film deposited on MNE surface after the modification of PVP and CTS, which made the electrode surface more rough. The existence of CTS/PVP could not only improve the conductivity, but also increase the specific surface area and active binding sites of the electrode. Importantly, it was also used as reducing agent for the selfassembly of AgNPs and adhesion agent for the immobilization of AgNPs. As shown in Fig. 1C and D, AgNPs were distributed uniformly on

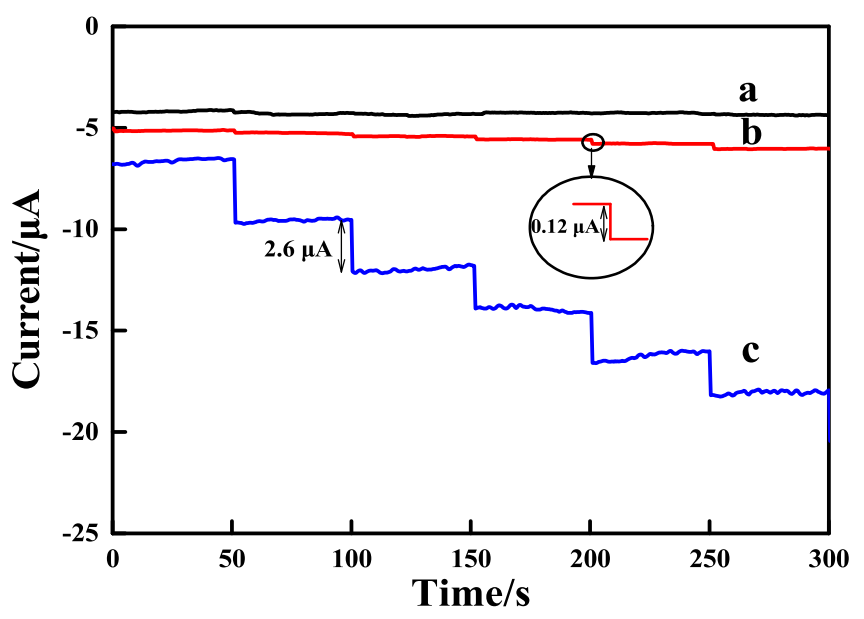

Fig. 2. Amperometric i-t curves of the bare MNE (a), CTS/PVP/MNE (b), and AgNPs/CTS/PVP/MNE (c) in $0.1 \mathrm{M} \mathrm{NaCl}$ solution with successive addition of the same concentration of $\mathrm{NO}_{3}^{-}$.

the surface of MNE with the diameter of about 150 to $400 \mathrm{~nm}$ after the self-assembly of AgNPs. AgNPs were successfully self-assembled and immobilized on the electrode because of the reduction capacity of CTS and strong affinity of PVP. The results of SEM characterization demonstrated that both CTS/PVP film and AgNPs were successfully modified on the MNE.

Subsequently, the AgNPs/CTS/PVP/MNE was characterized by means of XPS and CV. The XPS spectra of different electrodes were shown in Fig. S1. The presence of $\mathrm{N}$ and Ag signals proves the successful modification of CTS/PVP and AgNPs on the AgNPs/CTS/PVP/MNE. In addition, the Ag peaks of AgNPs/CTS/PVP/MNE shifted to higher binding energy, which might be caused by the strong interaction between the $\mathrm{O}$ of carbonyl group $(\mathrm{C}=\mathrm{O})$ in PVP and the AgNPs. The electrochemical properties of AgNPs/CTS/PVP/MNE were further investigated by CV (Fig. S2). The results showed that AgNPs and CTS/ PVP were modified successfully on MNE, which improved the conductivity and specific surface area of the electrode. The Electrode kinetics information was obtained by the CV behavior of AgNPs/CTS/PVP/MNE 


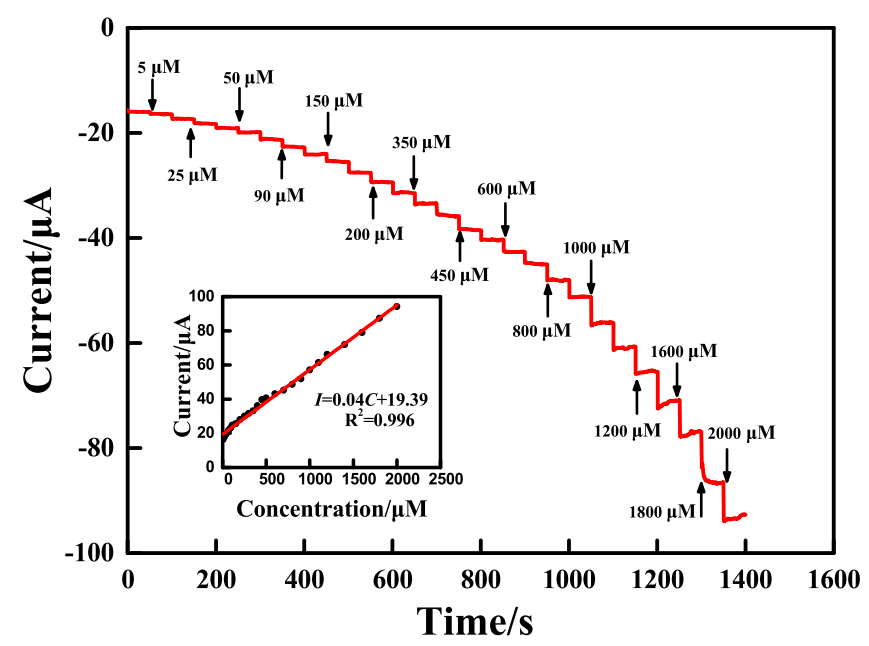

Fig. 3. Amperometric i-t curve obtained on the AgNPs/CTS/PVP/MNE with successive addition of $\mathrm{NO}_{3}^{-}$from 0.005 to $2 \mathrm{mM}$ in $0.1 \mathrm{M} \mathrm{NaCl}$ solution. Inset shows the corresponding calibration curve.

in $0.1 \mathrm{M} \mathrm{NaCl}$ solution containing $1 \mathrm{mM} \mathrm{NO}_{3}^{-}$at different scan rates (Fig. S3). The current response had a linear relationship with the square root of the scan rates, showing that the reduction of $\mathrm{NO}_{3}^{-}$on the AgNPs/ $\mathrm{CTS} / \mathrm{PVP} / \mathrm{MNE}$ was a diffusion-controlled electrode process.

\subsection{Electrochemical responses of AgNPs/CTS/PVP/MNE to $\mathrm{NO}_{3}^{-}$}

To explore the electrocatalytic reduction performance of CTS/PVP/ AgNPs/MNE to $\mathrm{NO}_{3}^{-}$, the current responses of MNE (a), CTS/PVP/MNE (b), and AgNPs/CTS/PVP/MNE (c) were compared by amperometric i-t method in $0.1 \mathrm{M} \mathrm{NaCl}$ solution with the potential of $-1.1 \mathrm{~V}$ (Fig. 2). It can be seen that there was no response signal of $\mathrm{NO}_{3}^{-}$could be obtained on the bare MNE. A much small cathodic response to $\mathrm{NO}_{3}^{-}(0.12 \mu \mathrm{A})$ could be detected by the CTS/PVP/MNE, which indicated CTS/PVP played a limited role for the amperometric detection of $\mathrm{NO}_{3}^{-}$. Although the promotion effect of CTS/PVP was limited, it provided a large specific surface area and improved conductivity. PVP has the advantages of high surface activity and strong adsorption capacity, which are helpful for the detection of $\mathrm{NO}_{3}^{-}$[35]. Compared to MNE and CTS/PVP/MNE, the AgNPs/CTS/PVP/MNE showed the best performance for $\mathrm{NO}_{3}^{-}$detection $(2.6 \mu \mathrm{A})$ thanks to the excellent catalytic performance of AgNPs. CTS can form stable conductive membrane with PVP and provide conditions for the self-assembly of AgNPs on the MNE. In addition, due to the strong affinity between AgNPs and pyridyl group of PVP which formed film with CTS, the stability of the AgNPs/CTS/PVP/MNE is significantly improved. So, the enhanced performance of AgNPs/CTS/PVP/MNE was caused by the combination of the excellent properties of CTS/PVP and AgNPs.

\subsection{Optimization for $\mathrm{NO}_{3}^{-}$determination with the AgNPs/CTS/PVP/ MNE}

The following parameters were optimized for the amperometric detection of $\mathrm{NO}_{3}^{-}$: mass ratio of CTS to PVP (Fig. S4A); deposition time of CTS/PVP (Fig. S4B); self-assembly time of the AgNPs (Fig. S4C); Respective data and Figures were given in the Supporting Information. The following experimental conditions were found to give best results: mass ratio of CTS to PVP (1:1); deposition time of CTS/PVP (400 s); selfassembly time of the AgNPs (5 min).

\subsection{Calibration curve}

Fig. 3 shows the calibration curve and corresponding amperometric i-t plots obtained at AgNPs/CTS/PVP/MNE in $0.1 \mathrm{M} \mathrm{NaCl}$ solution under
Table 1

Comparison of AgNPs/CTS/PVP/MNE with previously reported electrodes for $\mathrm{NO}_{3}^{-}$determination.

\begin{tabular}{|c|c|c|c|c|}
\hline Electrode & Method & $\begin{array}{l}\text { Linear range } \\
(\mathrm{mM})\end{array}$ & $\begin{array}{l}\text { Detection limit } \\
(\mu \mathrm{M})\end{array}$ & Ref. \\
\hline $\mathrm{Cu}$ & DPV & $0.1-2.5$ & 11.0 & [40] \\
\hline L-SCMNPs-CPES & SWV & $0.10-1.982$ & 87.0 & [41] \\
\hline $\begin{array}{l}\mathrm{Pd} / \mathrm{Sn} / \text { microband } \\
\text { electrode }\end{array}$ & LSV & $0.016-0.333$ & - & [42] \\
\hline $\begin{array}{l}\text { CNT-PPy-Pt } \\
\text { electrode }\end{array}$ & $\mathrm{CV}$ & $0.0005-0.01$ & 0.2 & [43] \\
\hline $\begin{array}{l}\text { AgNS on carbon and } \\
\text { Ag UMEs }\end{array}$ & $\mathrm{CV}$ & $0.004-1$ & $3.2-5.1$ & [44] \\
\hline $\begin{array}{l}\text { Nano-Ag/gold } \\
\text { electrode }\end{array}$ & $\mathrm{CV}$ & $0.2-1.4$ & 24 & [45] \\
\hline $\mathrm{Ag} / \mathrm{GCE}$ & $\mathrm{i}-\mathrm{t}$ & $0.01-5$ & 4 & {$[46]$} \\
\hline $\begin{array}{l}\text { AgNPs/CTS/PVP/ } \\
\text { MNE }\end{array}$ & i-t & $0.005-2$ & 1.2 & $\begin{array}{l}\text { This } \\
\text { work }\end{array}$ \\
\hline
\end{tabular}

L-SCMNPs, 3,6-bis(2-[2-sulfanyl-ethylimino-methyl]-4-(4-nitro-phenylazo)phenol)pyridazine coated $\mathrm{SiO}_{2} @ \mathrm{Fe}_{3} \mathrm{O}_{4}$; CPEs, carbon paste electrodes; CNT, carbon nanotubes; PPy, polypyrrole; AgNS, Ag nanostructures; UMEs, ultramicroelectrodes; GCE, glassy carbon electrode; DPV, differential pulse voltammetry; SWV, square wave voltammetry; LSV, linear sweep voltammogram.

the optimal conditions described above. The current response was linear with the concentration of $\mathrm{NO}_{3}^{-}$ranging from 0.005 to $2 \mathrm{mM}$. The regression equation was $I=0.04 C+13.39\left(\mathrm{R}^{2}=0.996\right)$, where $I$ represented the current in $\mu \mathrm{A}$, and $C$ was $\mathrm{NO}_{3}^{-}$concentration in $\mathrm{mM}$. The detection limit (LOD) of AgNPs/CTS/PVP/MNE for $\mathrm{NO}_{3}^{-}$determination was calculated as $1.2 \mu \mathrm{M}$ under the optimal conditions. The comparison of the AgNPs/CTS/PVP/MNE fabricated here with other electrodes published previously for $\mathrm{NO}_{3}^{-}$determination is presented in Table 1. It can be seen that when compared with other electrodes, the so-fabricated $\mathrm{AgNPs} / \mathrm{CTS} / \mathrm{PVP} / \mathrm{MNE}$ showed superior performance for $\mathrm{NO}_{3}^{-}$determination with higher sensitivity and wider linear range.

\subsection{Repeatability, selectivity and stability}

To investigate the suitability of the AgNPs/CTS/PVP/MNE for $\mathrm{NO}_{3}^{-}$ determination in complex water samples, its repeatability, selectivity and stability were all evaluated. The repeatability of the AgNPs/CTS/ $\mathrm{PVP} / \mathrm{MNE}$ was evaluated by detecting $1 \mathrm{mM} \mathrm{NO}_{3}^{-}$at the same electrode for fifty measurements, and the relative standard deviation (RSD) was calculated to be $3.1 \%$, indicating a good repeatability of the AgNPs/ CTS/PVP/MNE. The effects of possible interfering species for the amperometric determination of $\mathrm{NO}_{3}^{-}$were investigated by adding foreign species into the $0.1 \mathrm{M} \mathrm{NaCl}$ solution containing $1 \mathrm{mM} \mathrm{NO}_{3}^{-}$under the optimal conditions. The results showed that 30 -fold $\mathrm{Na}^{+}, \mathrm{Mg}^{2+}, \mathrm{Cr}^{3+}$ and $\mathrm{Cu}^{2+}, 10$-fold $\mathrm{Zn}^{2+}, \mathrm{As}^{3+}$ and 4-nitrophenol, and equivalent $\mathrm{Fe}^{2+}$ and $\mathrm{Cl}^{-}$did not affect the determination of $\mathrm{NO}_{3}^{-}(<5 \%$ of response current change). The CV curves of AgNPs/CTS/PVP/MNE in $1 \mathrm{mM} \mathrm{NO}_{3}^{-}$solution with and without typical reducible species including $\mathrm{Cu}^{2+}(30 \mathrm{mM})$ and 4-nitrophenol (10 mM) were presented in Fig. S5. These results showed that the AgNPs/CTS/PVP/MNE proposed here had a good selectivity for $\mathrm{NO}_{3}^{-}$determination. Additionally, the stability of the AgNPs/CTS/PVP/ MNE was investigated by measuring the current response of $1 \mathrm{mM} \mathrm{NO}_{3}^{-}$ every day with the same AgNPs/CTS/PVP/MNE. There was no obvious decrease of the current response after 15 days, indicating the good stability of the AgNPs/CTS/PVP/MNE.

\subsection{Practical application for $\mathrm{NO}_{3}^{-}$determination in seawater}

To evaluate the practical application of the so-fabricated AgNPs/ $\mathrm{CTS} / \mathrm{PVP} / \mathrm{MNE}$, it was used for the determination of $\mathrm{NO}_{3}^{-}$in real coastal seawater samples. In July 2019, seawater samples were collected at 9 stations at the Yellow River Estuary, DongYing, China. The sampling locations and corresponding latitude and longitude information of each 
Table 2

Comparison of the results for $\mathrm{NO}_{3}^{-}$determination in seawater samples by AgNPs/ CTS/PVP/MNE and ion chromatography (IC) method $(\mathrm{n}=3)$.

\begin{tabular}{lll}
\hline Seawater samples & Detected by this method $(\mu \mathrm{M})$ & Detected by IC $(\mu \mathrm{M})$ \\
\hline S1 & $143.8 \pm 4.6$ & 148.9 \\
S2 & $167.6 \pm 7.3$ & 163.4 \\
S3 & $241.2 \pm 10.5$ & 245.5 \\
S4 & $319.4 \pm 12.2$ & 318.6 \\
S5 & $276.2 \pm 11.7$ & 286.1 \\
S6 & $341.1 \pm 15.3$ & 338.8 \\
S7 & $328.2 \pm 13.6$ & 330.7 \\
S8 & $383.8 \pm 17.1$ & 392.0 \\
\hline
\end{tabular}

location was presented in Fig S6 and Table S1 respectively. After collection, the samples were filtered $(0.45 \mu \mathrm{m}$ membrane $)$ and kept in a refrigerator at $4{ }^{\circ} \mathrm{C}$ until detection. Before determination, all the samples were diluted ten times and determined directly without $\mathrm{pH}$ adjustment. The concentrations of $\mathrm{NO}_{3}^{-}$in the real seawater samples were determined by standard addition method. As shown in Table 2, the results obtained with the AgNPs/CTS/PVP/MNE were in agreement with that detected by ion chromatography (IC) method. The results indicated that the AgNPs/CTS/PVP/MNE fabricated here might be a reliable and suitable candidate for the direct determination of $\mathrm{NO}_{3}^{-}$in seawater.

\section{Conclusions}

In summary, a novel and effective AgNPs/CTS/PVP/MNE was fabricated for sensitive and direct determination of $\mathrm{NO}_{3}^{-}$in seawater. The CTS/PVP not only improved the conductivity and specific surface area of the MNE, but also provided the reduction conditions needed for $\mathrm{Ag}^{+}$ reduction and strong binding affinity for combination of AgNPs which had excellent catalytic activity for $\mathrm{NO}_{3}^{-}$. The combined outstanding properties of MNE, CTS/PVP and AgNPs made the AgNPs/CTS/PVP/ $\mathrm{MNE}$ a promising sensor for the direct determination of $\mathrm{NO}_{3}^{-}$in seawater.

\section{CRediT authorship contribution statement}

Jie Wang: Methodology, Writing - original draft. Ying Li: Methodology. Dawei Pan: Conceptualization. Haitao Han: Conceptualization, Writing - review \& editing. Peiqing Zhang: Writing - review \& editing.

\section{Declaration of Competing Interest}

The authors declare that they have no known competing financial interests or personal relationships that could have appeared to influence the work reported in this paper.

\section{Acknowledgments}

This work was financially supported by the National Key R\&D Program of China (2019YFD0901103), the Original Innovation Project of Chinese Academy of Sciences (ZDBS-LY-DQC009), and the Shandong Key Laboratory of Coastal Environmental Processes, YICCAS (2019SDHADKFJJ14).

\section{Appendix A. Supplementary data}

Supplementary data to this article can be found online at https://doi. org/10.1016/j.microc.2021.105965.

\section{References}

[1] M.J. Moorcroft, J. Davis, R.G. Compton, Detection and determination of nitrate and nitrite: a review, Talanta 54 (2001) 785-803.

[2] M. Sohail, S.B. Adeloju, Nitrate biosensors and biological methods for nitrate determination, Talanta 153 (2016) 83-98.
[3] J.-Z. Zhang, C.J. Fischer, A simplified resorcinol method for direct spectrophotometric determination of nitrate in seawater, Mar. Chem. 99 (1-4) (2006) 220-226.

[4] J. Du, Y. Fa, Y. Zheng, X. Li, F. Du, H. Yang, Measurement of trace nitrate concentrations in seawater by ion chromatography with valve switching, Chin. J. Ocean. Limn. 32 (3) (2014) 732-736.

[5] S.R. Carpenter, N.F. Caraco, D.L. Correll, Nonpoint pollution of surface waters by phosphorus and nitrogen, Ecol. Appl. 8 (1998) 559-568.

[6] M.I.H. Helaleh, T. Korenaga, Ion chromatographic method for simultaneous determination of nitrate and nitrite in human saliva, J. Chromatogr. B 744 (2) (2000) 433-437.

[7] M. Jimidar, C. Hartmann, N. Cousement, D.L. Massart, Determination of nitrate and nitrite in vegetables by capillary electrophoresis with indirect detection, J. Chromatogr. A 706 (1-2) (1995) 479-492.

[8] P. Raimbault, G. Slawyk, B. Coste, J. Fry, Feasibility of using an automated colorimetric procedure for the determination of seawater nitrate in the 0 to $100 \mathrm{nM}$ range: Examples from field and culture, Mar. Biol. 104 (2) (1990) 347-351.

[9] A. Sempere, J. Oliver, C. Ramos, Simple determination of nitrate in soils by secondderivative spectroscopy, Eur. J. Soil. Sci. 44 (1993) 633-639.

[10] R. Linker, M. Weiner, I. Shmulevich, Nitrate determination in soil pastes using attenuated total reflectance mid-infrared spectroscopy: improved accuracy via soil identification, Biosyst. Eng. 94 (2006) 111-118.

[11] J.R. Thayer, R.C. Huffaker, Determination of nitrate and nitrite by high-pressure liquid chromatography: comparison with other methods for nitrate determination, Anal. Biochem. 102 (1980) 110-119.

[12] Y. Li, H. Han, D. Pan, P. Zhang, Fabrication of a micro-needle sensor based on copper microspheres and polyaniline film for nitrate determination in coastal river waters, J. Electrochem. Soc. 166 (12) (2019) B1038-B1043.

[13] A. Nezamzadeh-Ejhieh, Z. Nematollahi, Surfactant modified zeolite carbon paste electrode (SMZ-CPE) as a nitrate selective electrode, Electrochim. Acta 56 (24) (2011) 8334-8341.

[14] J. Davis, M.J. Moorcroft, S.J. Wilkins, Electrochemical detection of nitrate and nitrite at a copper modified electrode, Analyst 125 (2000) 737-741.

[15] G. Denuault, The contribution of microelectrodes to electroanalytical chemistry: from reaction mechanisms and scanning electrochemical microscopy to ocean sensors, Isr. J. Chem. 50 (2010) 374-381.

[16] G.E. Dima, A.C.A. de Vooys, M.T.M. Koper, Electrocatalytic reduction of nitrate at low concentration on coinage and transition-metal electrodes in acid solutions, J. Electroanal. Chem. 554-555 (2003) 15-23.

[17] S. Piao, Y. Kayama, Y. Nakano, K. Nakata, Y. Yoshinaga, K. Shimazu, Nitrate reduction on tin-modified rhodium, ruthenium, and iridium electrodes, J. Electroanal. Chem. 629 (1-2) (2009) 110-116.

[18] A. Anastasopoulos, L. Hannah, B.E. Hayden, High throughput optimisation of PdCu alloy electrocatalysts for the reduction of nitrate ions, J. Catal. 305 (2013) 27-35.

[19] H.-L. Li, J.Q. Chambers, D.T. Hobbs, Electroreduction of nitrate ions in concentrated sodium hydroxide solutions at lead, zinc, nickel and phthalocyaninemodified electrodes, J. Appl. Electrochem. 18 (3) (1988) 454-458.

[20] M.E. Bodini, D.T. Sawyer, Voltammetric determination of nitrate ion at parts-perbillion levels, Anal. Chem. 49 (3) (1977) 485-489.

[21] M. Shibata, Electrochemical synthesis of urea at gas-diffusion electrodes, J. Electrochem. Soc. 145 (1998) 2348-2353.

[22] P.G. Fenga, N.R. Stradiotto, M.I. Pividori, Silver nanocomposite electrode modified with hexacyanoferrate. Preparation, characterization and electrochemical behaviour towards substituted anilines, Electroanalysis 23 (5) (2011) 1100-1106.

[23] S. Maheswari, P. Sridhar, S. Pitchumani, Carbon-supported silver as cathode electrocatalyst for alkaline polymer electrolyte membrane fuel cells, Electrocatalysis 3 (1) (2012) 13-21.

[24] C.-L. Lee, H.-P. Chiou, C.-M. Syu, C.-C. Wu, Silver triangular nanoplates as electrocatalyst for oxygen reduction reaction, Electrochem. Commun. 12 (11) (2010) 1609-1613.

[25] F. Manea, A. Remes, C. Radovan, R. Pode, S. Picken, J. Schoonman, Simultaneous electrochemical determination of nitrate and nitrite in aqueous solution using $\mathrm{Ag}$ doped zeolite-expanded graphite-epoxy electrode, Talanta 83 (1) (2010) 66-71.

[26] J.-G. Lin, W.-L. Chen, Acupuncture analgesia: a review of its mechanisms of actions, Am. J. Chin. Med. 36 (04) (2008) 635-645.

[27] J.G. Lin, W.L. Chen, Review: acupuncture analgesia in clinical trials, Am. J. Chin. Med. 37 (2009) 1-18.

[28] Y. Li, L. Tang, Y. Ning, In vivo monitoring of serotonin by nanomaterial functionalized acupuncture needle, Sci. Rep. 6 (2016) 28018.

[29] H. Han, W. Tao, X. Hu, X. Ding, D. Pan, C. Wang, S. Xu, Needle-shaped electrode for speciation analysis of copper in seawater, Electrochim. Acta 289 (2018) 474-482.

[30] F.J. Pavinatto, L. Caseli, O.N. Oliveira Jr., Chitosan in nanostructured thin films, Biomacromolecules 11 (8) (2010) 1897-1908.

[31] M.M. Abdi, L.C. Abdullah, A.R. Sadrolhosseini, Surface plasmon resonance sensing detection of mercury and lead ions based on conducting polymer composite, Plos One 6 (2011), e24578.

[32] A.G. Yavuz, A. Uygun, H. Kaplan Can, The effect of synthesis media on the properties of substituted polyaniline/chitosan composites, Carbohyd. Res. 346 (14) (2011) 2063-2069.

[33] L.i. Wang, H. Zhu, H. Hou, Z. Zhang, X. Xiao, Y. Song, A novel hydrogen peroxide sensor based on Ag nanoparticles electrodeposited on chitosan-graphene oxide/ cysteamine-modified gold electrode, J. Solid State Electrochem. 16 (4) (2012) 1693-1700. 
[34] M. Wan, Z. Liu, S. Li, B. Yang, W. Zhang, X. Qin, Z. Guo, Silver nanoaggregates on chitosan functionalized graphene oxide for high-performance surface-enhanced Raman scattering, Appl. Spectrosc. 67 (7) (2013) 761-766.

[35] S. Zhang, Z. Shi, J. Wang, Sensitive and rapid determination of quinoline yellow in drinks using polyvinylpyrrolidone-modified electrode, Food Chem. 173 (2015) 449-453.

[36] T. Balankura, How structure-directing agents control nanocrystal shape: PVPmediated growth of Ag nanocubes, Nano. Lett. 15 (2015) 7711-7717.

[37] R. Zhao, S. Sun, W. Hao, H. Guo, Y. Gao, L. Shi, A highly sensitive determination for the melamine in milk on MIL-101/AuNPs/CTS-PVP-rGO/GCE electrochemical sensor, Russ. J. Electrochem. 55 (7) (2019) 651-662.

[38] A. Pavinatto, L.A. Mercante, M.H.M. Facure, R.B. Pena, R.C. Sanfelice, L.H. C. Mattoso, D.S. Correa, Ultrasensitive biosensor based on polyvinylpyrrolidone/ chitosan/reduced graphene oxide electrospun nanofibers for $17 \alpha$ - Ethinylestradiol electrochemical detection, Appl. Surface Sci. 458 (2018) 431-437.

[39] H. Han, D. Pan, Y. Li, Stripping voltammetric determination of lead in coastal waters with a functional micro-needle electrode, Fron. Mar. Sci. 7 (2020) 196.

[40] T.R.L.C. Paixão, J.L. Cardoso, M. Bertotti, Determination of nitrate in mineral water and sausage samples by using a renewable in situ copper modified electrode, Talanta 71 (1) (2007) 186-191.
[41] A. Afkhami, T. Madrakian, H. Ghaedi, H. Khanmohammadi, Construction of a chemically modified electrode for the selective determination of nitrite and nitrate ions based on a new nanocomposite, Electrochim. Acta 66 (2012) 255-264.

[42] Y. Fu, C. Bian, J. Kuang, A palladium-tin modified microband electrode array for nitrate determination, Sensors 15 (2015) 23249-23261.

[43] T. Madasamy, M. Pandiaraj, M. Balamurugan, K. Bhargava, N.K. Sethy, C. Karunakaran, Copper, zinc superoxide dismutase and nitrate reductase coimmobilized bienzymatic biosensor for the simultaneous determination of nitrite and nitrate, Biosens. Bioelectron. 52 (2014) 209-215.

[44] H.R. Zhad, R.Y. Lai, Comparison of nanostructured silver-modified silver and carbon ultramicroelectrodes for electrochemical detection of nitrate, Anal. Chim. Acta. 892 (2015) 153-159.

[45] A.J. Gross, S. Holmes, S.E.C. Dale, Nitrite/nitrate detection in serum based on dualplate generator-collector currents in a microtrench, Talanta 131 (2015) 228-235.

[46] L. Guadagnini, D. Tonelli, Carbon electrodes unmodified and decorated with silver nanoparticles for the determination of nitrite, nitrate and iodate, Sens. Actuators B Chem. 188 (2013) 806-814. 